Original Research Paper

\title{
Influence of Age and Gender on Mandible Among South Indian Population
}

\author{
Kathirvelu Dhandapani and Anburajan Mariamichael \\ Department of Biomedical Engineering, SRM University, Kattankulathur-603 203, Tamil Nadu, India
}

\author{
Article history \\ Received: 15-09-2014 \\ Revised: 28-11-2014 \\ Accepted: 12-03-2015 \\ Corresponding Author: \\ M. Anburajan \\ Department of Biomedical \\ Engineering, \\ SRM University, \\ Kattankulathur-603 203, Tamil \\ Nadu, India \\ Email: anbufelix@gmail.com
}

\begin{abstract}
The decrease of bone strength on ageing is a globally observed phenomenon among all human beings. The association between oral health and bone health has not been much researched among the Indian population. So, the proposed study was aimed to extract information about mandibular indices from dental panoramic radiographs and to identify its age and gender related changes among the south Indian population. The study involved 279 subjects, which included 103 men $(46 \pm 11.55$ years) and 176 women (47.2 \pm 12.49 years). The panoramic radiographs were obtained from a digital orthopantomogram scanner. The subjects were categorised into various decade age sub-groups for both the genders: 31-40, 41-50, 51-60, 61-70 and 71-80 years. The subjects were also further classified into three groups as: Normal, mild and severe based on the erosion of the endosteal margin of mandible cortical bone. The measurement of the Mandibular Cortical Thickness (MCT), Panoramic Mandibular Index (PMI) and assessment of the endosteal margin of the mandibular cortical bone was performed on the radiograph. The ANOVA test revealed a statistically significant difference for the MCT and PMI as $(F=6.98 ; \mathrm{p}<0.001)$ and $(\mathrm{F}=4.49 ; \mathrm{p}<0.01)$ respectively between subjects of the various decade age sub-groups. The Chi-square tests revealed higher levels of significances $(p<0.001)$ for the variations in the endosteal margin with age. The results of the study conclude that the MCT, PMI and the endosteal margin of mandible are age depended. The decrement of radiomorphometric indices was more prominent in females than males and they exponentially decreased after the sixth decade of age for both the genders. The MCT and PMI decreased by 6.41 and $4.8 \%$ respectively for males and by 23.2 and $30.8 \%$ respectively for females in the older groups.
\end{abstract}

Keywords: Dental Panoramic Radiographs, Bone Strength, Mandible Cortical Thickness, Endosteal Margin, Panoramic Mandibular Index, Indian Population, Radiomorphometric Indices

\section{Introduction}

In vertebrates, bones play an important function of providing mechanical support and structure and thereby enabling mobility. Bones are a storehouse of vital minerals and provide protection to heart, lungs, brain and other organs in human beings (Taichman, 2005). The onset of bone decrementation generally occurs at about the third decade of life. Although loss of bone mass is common for both men and women on ageing, women tend to lose bone mass, at a greater rate than men especially after menopause (NIH, 2004). Peak bone mass is referred to as the maximum bone mass attained in a person's life indicating the growth process of bones are completed. The age of attaining peak bone mass varies in different parts of the skeletal structure and for populations across the globe. The factors that determine the peak bone mass are: Genetic, nutrition, physical activity and disease influencing bone development. The rate of bone loss depends on race, gender, geographical location and age. Studies have proven that black women possess superior bone quality than Asian counterparts and seldom incur severe bone loss (Sapthagirivasan and Anburajan, 2012). Though the loss of bone quality on ageing is observed in men, the severity is minimal compared to equivalent females. The loss of bone quality that supports the jaw and anchoring the teeth can lead to loose teeth, tooth loss, gums detaching from the teeth or receding gums and poor fitting dentures. It's been reported that women with poor bone quality incur severe tooth loss 
than normal women (OHBD, 2012). Therefore, dentists could be in a unique position to identify people with low bone quality at the earliest as many visit their dentist frequently than their doctor. The impact of the bone related disorders can be examined by several imaging modalities: Radiography, Quantitative Computed Tomography (QCT), Dual-energy X-ray Absorptiometry (DXA), etc. Even though DXA has been regarded as the World Health Organization (WHO) standard method for Bone Mineral Density (BMD) measurement, it involves huge capital investment and treatment cost. It has been reported that the number of DXA machines available in India is 250 i.e., about 0.2 DXA machines per million populations, which falls well below the threshold, recommended by European regulations, pertaining to DXA machine (IOF, 2009). Studies by researchers on mandibular bone reveal that there exists a strong relationship between oral health and bone strength (Taguchi, 2010; Karayianni et al., 2007; Alman et al., 2012; Benson et al., 1991; Khojastehpour et al., 2009). The effect of deterioration of skeletal bone mass influencing morphological changes in mandible has been investigated among population all over the world (Krejci and Bissada, 2002; Jonasson et al., 2001; Jeffcoat et al., 2000; Klemetti and Vainio, 1993; Klemetti et al., 1994). The methods for screening individuals with low bone mass from Dental Panoramic Radiographs (DPR) are simple, cost effective and noninvasive technique (Ledgerton et al., 1997). Therefore, the panoramic measurements can also be considered as a reference tool to identify individuals who are at risk of possessing low bone mass and recommend them to undergo further advanced investigation.

Dentists are accustomed to the use of DPR for their routine dental Examinations to examine the oral cavities and jaws. Studies on the DPR reveal that mandible radiomorphometric indices like the Mandible Cortical Thickness (MCT), the morphology of endosteal margin and the Panoramic Mandibular Index (PMI) could be used to identify individuals at the risk of low BMD and to prescribe them to undergo DXA scan for BMD assessment. It has been found that the MCT on a DPR significantly correlated with DXA-BMD in the hip, lumbar spine and forearm (Karayianni et al., 2007; Alman et al., 2012; Benson et al., 1991). Hence, measurement of the MCT could be considered as an effective method for screening people who are at risk of low bone strength. Studies have reported that the PMI calculated, as per the protocol detailed by (Benson et al., 1991) could also be used to discriminate between normal and low bone strength group. Klemetti and Vainio (1993; Klemetti et al., 1994) demonstrated that patterns of intracortical resorption of the mandibular cortex showed variations for subjects of normal and low bone mass group.

The adequacy of knowledge on the relationship between oral health and bone health among the Indian population has been limited. The extent of mandibular indices has not been well documented among the Indian population. So, the proposed study was aimed to extract information about mandibular indices from DPR and to identify its age and gender related variations in the south Indian population.

\section{Materials and Methods}

\section{Study Population}

The DPR's were collected from adults aged greater than 30 years, who visited the dental clinic in the SRM medical college hospital and research Centre between March 2013 and August 2013. The Institutional ethical committee approved the study protocol and a written informed consent in their own language was obtained from the subjects who were willing to take part in the study. Post-menopausal women undergoing hormone replacement therapy as well as calcium supplement therapy, subjects who underwent hysterectomy or oophorectomy, subjects with fractures, deformities and suffering from bone related disorders were not included in the study. The study involved 279 subjects which included 103 men (mean age: $46 \pm 11.55$ years) and 176 women (mean age: $47.2 \pm 12.49$ years).

\section{Radiographic Analyses}

The radiographs were taken using a digital Orthopantomogram scanner (KODAK 8000C) at 70KV by a trained radiographer following the standard procedure and Digital Imaging and Communications in Medicine (DICOM) viewer recommended by the manufacturer was used to analyse the radiographs. The DPR was assessed independently by two authors, who were trained by a radiologist possessing 15 years of experience to identify the region of interest, location of the mental foramen on both sides and locating the inner and outer boundaries of mandibular cortex.

The MCT was measured on both the sides at the site of mental foramen as shown in the Fig. 1. On locating the mental foramen, a line was drawn passing through the center of mental foramen perpendicular to the tangent drawn to the lower border of the mandibular cortex on the digital radiograph. The measurement of the MCT was performed along this line on the radiograph.

The PMI was obtained by dividing the MCT by the distance between the center of mental foramen and the lower border of the mandibular cortex as proposed by (Benson et al., 1991) protocol. The measurement of MCT and PMI was performed bilaterally and average of each value was considered. An inter-rater agreement with a kappa coefficient of 0.9 was found between the measurements carried out by the two authors. The visual assessment of endosteal margin of the mandible cortical bone was performed and the radiographs were scored according to Klemetti index as normal, mild and severe as shown in Fig. 2. 


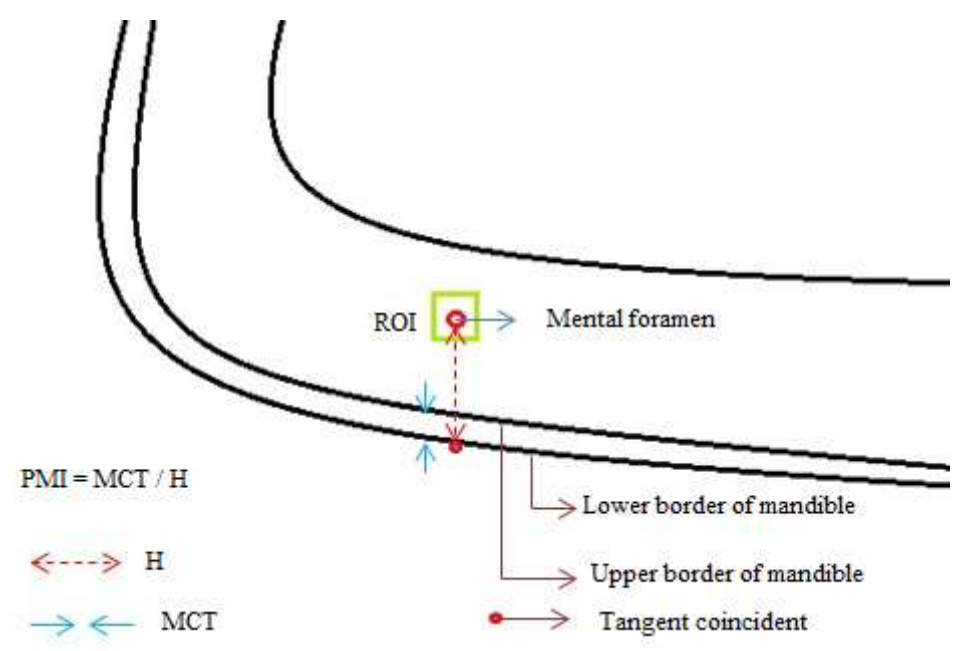

Fig. 1. Measurement of MCT and PMI on DPR

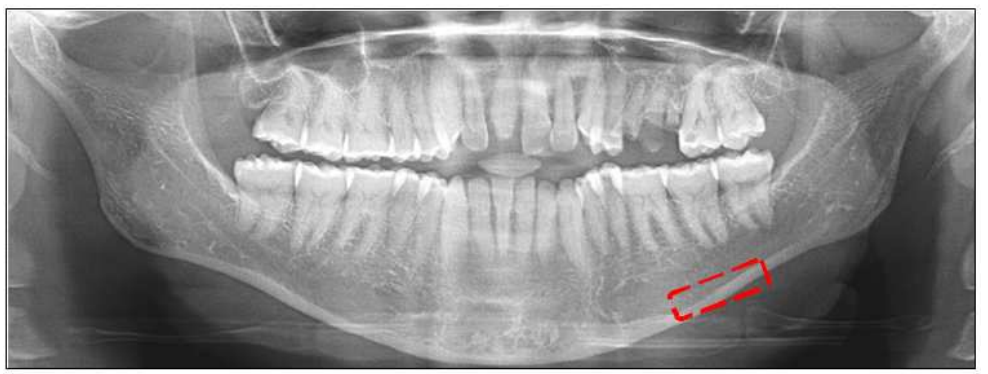

(a)

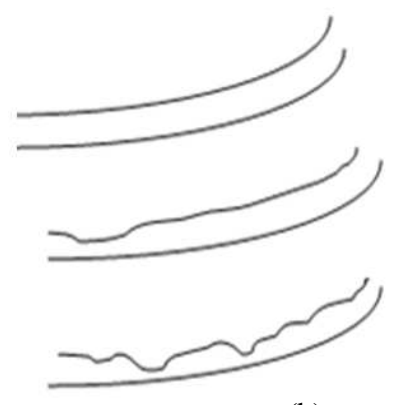

(b)
Normal

Mild

Severe
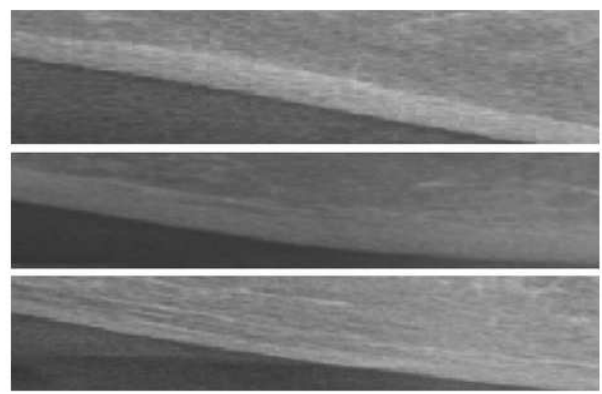

(c)

Fig. 2. (a) Input radiograph with marked endosteal region (b) Classification of endosteal margin (c) Illustration of (b) on DPR

\section{Statistical Analyses}

The study was carried out by dividing the subjects into five decade age categories for both genders: (a) 31-40 years, (b) 41-50 years, (c) 51-60 years, (d) 61-70 years and (e) 7180 years. The subjects were also further classified into three groups as: Normal, mild and severe based on the extent of erosion of the endosteal margin of mandible cortical bone.

The ANOVA was used for comparing and calculating mean PMI and MCT values for all categories. Post-hoc tests according to the Tukey HSD test was performed to determine group differences. The Chi-square test was performed to study the association between endosteal margin of mandible cortical bone with age. The data obtained were assessed by SPSS software package version 17.0 (SPSS Inc., Chicago, USA) and Microsoft Excel version 2007 (MS-Office package, Microsoft Inc., Redmond, USA).

\section{Results}

The data obtained were analysed and the following inferences were made. Out of the total subjects studied, $37 \%$ were men and $63 \%$ were women residing in a south Indian neighborhood. 


\section{Influence of Age}

When decade age groups of the entire study population were taken into consideration, the MCT and PMI decreased from the sixth decade of life and progressively decreased thereafter as shown in Table 1. The ANOVA test revealed a statistically significant difference for the MCT and PMI as $(\mathrm{F}=6.98 ; \mathrm{p}<0.001)$ and $(\mathrm{F}=4.49 ; \mathrm{p}<0.01)$ respectively between subjects of the various decade age groups. The Post-hoc tests using the Tukey HSD demonstrated statistically significant difference $(p<0.05)$ for the MCT between subjects aged greater than 70 years and subjects aged lesser than 70 years, whereas the PMI also exhibited statistical difference $(\mathrm{p}<0.05)$ between subjects aged greater than 70 and lesser aged groups.

When the influence of ageing on the endosteal margin of the cortical bone was taken into consideration, it was observed that there is steep rise on the severity of cortical bone erosion on ageing. This clearly implies that the onset of bone decrementation is associated with ageing. The Chisquare tests revealed higher level of significances $(p<0.001)$ between various age subgroups and variations in endosteal margin of cortical bone as shown in Table 2.

\section{Influence of Gender}

When only female subjects were taken into consideration, the ANOVA test revealed that a statistically significant difference for the MCT $(\mathrm{F}=9.01 ; \mathrm{p}<0.001)$ and PMI $(\mathrm{F}=7.1 ; \mathrm{p}<0.001)$ exits between subjects of the various decade age groups as shown in Table 3. The Posthoc tests using the Tukey HSD demonstrated statistically significant difference $(p<0.05)$ for the MCT between subjects aged greater than 60 years and subjects aged lesser than 60 years, Whereas the PMI also exhibited similar statistical significance at the level $(\mathrm{p}<0.05)$ between subjects aged greater than 70 years and those in the lesser aged group. When such comparison was performed among male population, it was observed that no such statistical significance found to exist for the various age groups as observed among their female counterparts.

Table 4 reveals the comparison performed between male and equivalent female age subgroups. The t-test revealed that the MCT exhibited high level of statistical significance at level $(\mathrm{p}<0.001)$ only at the older age sub-groups (61-70 years), whereas such significant differences were not observed in the remaining groups.

Table 1. Decade age group analysis of the entire study population

\begin{tabular}{|c|c|c|c|c|c|c|c|}
\hline \multirow{2}{*}{$\begin{array}{l}\text { Morphometric } \\
\text { measures }\end{array}$} & \multicolumn{5}{|c|}{ Age group (years) } & \multirow[b]{2}{*}{$\mathrm{F}$} & \multirow[b]{2}{*}{$\mathrm{p}$ value } \\
\hline & $31-40(n=103)$ & $41-50(n=74)$ & $51-60(n=64)$ & $61-70(n=26)$ & $71-80(\mathrm{n}=12)$ & & \\
\hline MCT (mm) & $2.89 \pm 0.43^{\mathrm{c}}$ & $2.79 \pm 0.48^{\mathrm{bc}}$ & $2.89 \pm 0.43^{\mathrm{c}}$ & $2.55 \pm 0.48^{\mathrm{ab}}$ & $2.31 \pm 0.56^{\mathrm{a}}$ & 6.977 & $<0.001^{* *}$ \\
\hline PMI & $0.24 \pm 0.05^{\mathrm{b}}$ & $0.23 \pm 0.05^{\mathrm{b}}$ & $0.23 \pm 0.05^{\mathrm{b}}$ & $0.21 \pm 0.03^{\mathrm{ab}}$ & $0.19 \pm 0.04^{\mathrm{a}}$ & 4.486 & $0.002^{* * *}$ \\
\hline
\end{tabular}

Note: $1{ }^{* *}$ denotes significance at $1 \%$ level

2. Different alphabets between age groups denote significance at 5\% level using Tukey HSD test

Table 2. Influence of age on endosteal margin of mandible

\begin{tabular}{|c|c|c|c|c|c|c|}
\hline \multirow[b]{2}{*}{ Age group } & \multicolumn{3}{|c|}{ Endosteal margin } & \multirow[b]{2}{*}{ Total } & \multirow[b]{2}{*}{ Chi-square value } & \multirow[b]{2}{*}{$\mathrm{p}$ value } \\
\hline & Normal & Mild & Severe & & & \\
\hline $31-40$ & $35-34$ [54] & $66-64$ [34] & $2-2[9]$ & 103 & 50.463 & $<0.001^{* * *}$ \\
\hline $41-50$ & $19-26$ [29] & $51-69[27]$ & $4-5$ [18] & 74 & & \\
\hline $51-60$ & $7-11[11]$ & $51-80[27]$ & $6-9$ [27] & 64 & & \\
\hline $61-70$ & $2-8[3]$ & $20-77$ [10] & $4-15$ [18] & 26 & & \\
\hline $71-80$ & $2-17$ [3] & $4-33$ [2] & $6-50$ [27] & 12 & & \\
\hline
\end{tabular}

Note: 1 . The value within () refers to row percentage

2 . The value within [] refers to column percentage

3. ${ }^{* * *}$ Denotes significant at $1 \%$ level

Table 3. Gender based decade age group analysis

\begin{tabular}{|c|c|c|c|c|c|c|c|c|}
\hline \multirow[b]{2}{*}{ Gender } & \multirow{2}{*}{$\begin{array}{l}\text { Morphometric } \\
\text { measures }\end{array}$} & \multicolumn{5}{|c|}{ Age group (years) } & \multirow[b]{2}{*}{$\mathrm{F}$} & \multirow[b]{2}{*}{$\mathrm{p}$ value } \\
\hline & & $31-40$ & $41-50$ & $51-60$ & $61-70$ & $71-80$ & & \\
\hline \multirow[t]{2}{*}{ Male } & MCT (mm) & $2.81 \pm 0.35$ & $2.76 \pm 0.41$ & $2.84 \pm 0.31$ & $2.85 \pm 0.3$ & $2.63 \pm 0.53$ & 0.322 & 0.863 \\
\hline & PMI & $0.21 \pm 0.04$ & $0.21 \pm 0.05$ & $0.21 \pm 0.03$ & $0.21 \pm 0.03$ & $0.2 \pm 0.03$ & 0.053 & 0.995 \\
\hline \multirow[t]{2}{*}{ Female } & $\mathrm{MCT}(\mathrm{mm})$ & $2.93 \pm 0.47^{b}$ & $2.8 \pm 0.52^{\mathrm{b}}$ & $2.91 \pm 0.48^{b}$ & $2.24 \pm 0.45^{\mathrm{a}}$ & $2.25 \pm 0.57^{\mathrm{a}}$ & 9.008 & $<0.001^{* *}$ \\
\hline & PMI & $0.26 \pm 0.05^{\mathrm{c}}$ & $0.24 \pm 0.05^{b c}$ & $0.24 \pm 0.05^{\mathrm{bc}}$ & $0.2 \pm 0.03^{\mathrm{ab}}$ & $0.18 \pm 0.04^{\mathrm{a}}$ & 7.096 & $<0.001^{* *}$ \\
\hline
\end{tabular}

Note: $1 .{ }^{* * *}$ denotes significance at $1 \%$ level

1. Different alphabets between age groups denote significance at $5 \%$ level using Tukey HSD test 
Table 4. Intergroup comparison between male and equivalent female decade agesub-groups

\begin{tabular}{llll}
\hline Age group (years) & Morphometric measures & t- value & P value \\
\hline $31-40$ & MCT (mm) & 1.356 & 0.18 \\
& PMI & 5.086 & $<0.001^{* *}$ \\
$41-50$ & MCT (mm) & 0.317 & 0.75 \\
& PMI & 2.496 & 0.02 \\
$51-60$ & MCT (mm) & 0.599 & 0.551 \\
& PMI & 2.323 & 0.02 \\
$61-70$ & MCT (mm) & 4.078 & $<0.001^{* *}$ \\
& PMI & 0.816 & 0.422 \\
\hline
\end{tabular}

Note: ${ }^{* * *}$ Denotes significant at $1 \%$ level

The PMI exhibited higher level of significance $(\mathrm{p}<0.001)$ for 31-40 and 61-70 age sub-groups, whereas other age subgroups 41-50, 51-60 showed statistical significance at the level $(\mathrm{p}<0.05)$. It is clearly evident that cortical thickness of mandible greatly diminishes on ageing resulting in low levels of MCT and PMI.

\section{Discussion}

The DPR are preferred by dentists to examine oral cavity and jaws of an individual more often than any other organ in the human body. Even though DXA has been considered as the WHO standard to measure BMD and diagnose osteoporosis, studies on the use of panoramic radiographs to predict BMD, suggest that the DPR's can be used to determine the loss of BMD in subjects.

The association of oral hygiene with bone health has well documented by several authors in literature (Taguchi, 2010; Karayianni et al., 2007; Alman et al., 2012; Benson et al., 1991; Khojastehpour et al., 2009). It has been studied that cortical changes of the mandible reflect the changes in the BMD of the skeleton. Studies have revealed that the MCT measured on DPR could be useful to identify people with low BMD (Karayianni et al., 2007; Devlin and Horner, 2002).

Devlin and Horner (2002) suggested that the panoramic radiographs can be used to generate a cost effective screening tool to predict the risk of low BMD and provide information about the bone quality of mandible as they noticed a considerable relationship between the MCT and BMD. The possible risk of osteoporosis could be diagnosed by observing the amount of tooth loss, weakening of mandible cortical bone and variations in the morphology of endosteal margin. In the present study it was noticed that there was a negative correlation between the MCT $(r=-0.22$; $\mathrm{p}<0.001)$ and PMI $(r=-0.23 ; \mathrm{p}<0.001)$ with age. The observations matched with the results of the study carried out by (Ledgerton et al., 1997). Earlier studies by (Ledgerton et al., 1999) among British women also reported that there was a steep fall in the MCT and PMI with age and the fall observed to be critical, by the beginning of sixth decade. Higher significances $(p<0.001)$ for cortical indices were observed between younger and older population and similar levels of significances were observed for mean PMI values for younger and older age groups. In the present study, similar worthwhile observations were made, where in-which, the MCT and PMI demonstrated a statistically significant difference of $(F=9.71 ; p<0.001)$ with respect to old and young aged groups and $(\mathrm{F}=14.55 ; \mathrm{p}<0.001)$ with respect to old and middle age groups among the studied population. Wherever there was significant reduction in the values of the MCT and PMI; it would be apparent due to ageing. The outcomes also correlated with the study conducted on Brazilian population by (Alonso et al., 2011) that the decrement in radiomorphometric indices for both the sexes are age dependent.

Estera et al. (2008) identified among the Lithuanian population that if the MCT was low, there is a tendency of high probability of osteoporosis and a strong relationship was noticed between increased risk of osteoporosis with corresponding decrease in the MCT in the mental region. In the current study, it is evident that an ageing population has reduced MCT and PMI, thereby providing an alarm that they might be at possible risk of low BMD.

The endosteal margin of cortical bone categorised based on Klemetti's index and MCT can be used for assessment of bone quality of the mandible. As DPR's are being widely used in dentistry, they could be successfully deployed for assessment of bone quality of the mandible.

Halling et al. (2005) demonstrated that endosteal margin of mandible cortical bone could be used as a valid tool to screen people with low BMD. In the present study, it was observed that the erosion of endosteal margin was predominant among ageing population, suggesting that ageing to be an important factor for low BMD. The findings indicate that dentists are in a position to identify the subjects and recommend those with positive findings for further assessment for the potential diagnosis of low BMD. Earlier studies have reported that visual assessment of endosteal margin has been utilised for identifying people with low bone quality has limited accuracy. Furthermore Horner et al. (2007) suggested that the MCT can perform better than visual assessment of DPR and also (Damilakis and Vlasiadis, 2011) supported that the MCT could perform better than PMI in distinguishing people with low BMD from normal subjects. 
Hence, the study highlights the importance of the usage of the various morphometric measurements to assess the cortical bone mass in an easy and cost effective manner and thereby to spot individuals with low bone mass.

The limitation of the present study is that the size of the study population is relatively less and comparison of results against a standard method has not been performed.

\section{Conclusion}

we noticed that the controlling factor for the studied population to be age with respect to MCT, PMI and the endosteal margin of mandible cortical bone. The radiomorphometric indices exhibited eminent decrementation after the sixth decade of life for both the genders. The MCT and PMI decreased in the older group with respect to the younger group by 6.41 and $4.8 \%$ respectively for males and by 23.2 and $30.8 \%$ respectively in females. The deterioration was very prominent in females than males with reference to radiomorphometric indices.

\section{Acknowledgment}

The authors wish to express their deep sense of gratitude to the management of SRM Hospital and Research Centre for providing the required facilitating infrastructure. They also wish to thank Mr. V. Sapthagirivasan, Research Scholar, for his kind support and help.

\section{Funding Information}

All costs and related expenditures have been managed by the first author only.

\section{Author's Contributions}

Kathirvelu Dhandapani: The design, implementation, statistical analysis and drafting the manuscript.

Anburajan Mariamichael: Guided the entire study by giving technical support, conceptual advice and proof reading the article.

\section{Ethics}

The Institutional Ethical Committee approved the study protocol and written informed consent was obtained from all participants.

\section{References}

Alman, A.C., L.R. Johnson, D.C. Calverley, G.K. Grunwald and D.C. Lezotte et al., 2012. Diagnostic capabilities of fractal dimension and mandibular cortical width to identify men and women with decreased bone mineral density. Osteoporosis Int., 23: 1631-1636. DOI: 10.1007/s00198-011-1678-y
Alonso, M.B.C.C., A.R.G. Cortes, J.C. Angela, S.A. Emiko and F. Haiter-Neto et al., 2011. Assessment of panoramic radiomorphometric indices of the mandible in a Brazilian population. ISRN Rheumatol. DOI: 10.5402/2011/854287

Benson, B.W., T.J. Prihoda and B.J. Glass, 1991. Variations in adult cortical bone mass as measured by a panoramic mandibular index. Oral Surgery, Oral Medicine Oral Pathol., 71: 349-356. DOI: 10.1016/0030-4220(91)90314-3

Damilakis, J. and K. Vlasiadis, 2011. Have panoramic indices the power to identify women with low BMD at the axial skeleton? Eur. J. Med. Phys., 27: 39-43. DOI: 10.1016/j.ejmp.2010.03.002

Devlin, H. and K. Horner, 2002. Mandibular radiomorphometric indices in the diagnosis of reduced skeletal bone mineral density. Osteoporosis Int., 13: 373-378. PMID: 12086347

Estera, M., A. Vidmantas, P. Vytaute, T. Marija and M. Rasmute, 2008. Relationship between mandibular cortical bone height and bone mineral density of lumbar spine. Stomatologija, Baltic Dental Maxillofacial J., 10: 72-75. PMID: 18708740

Halling, A., R. Persson, J. Berglund, O. Johansson and S. Renvert, 2005. Comparison between the Klemetti index and heel DXA BMD measurements in the diagnosis of reduced skeletal bone mineral density in the elderly. Osteoporos Int., 16: 999-1003. PMID: 15605191

Horner, K., K. Karayianni, A. Mitsea, L. Berkas and M. Mastoris et al., 2007. The mandibular cortex on radiographs as a tool for osteoporosis risk assessment: The OSTEODENT project. J. Clin. Dens., 10: 138-146. PMID: 15605191

IOF, 2009. The Asian Audit Epidemiology, costs and burden of osteoporosis in Asia. International Osteoporosis Foundation.

Jeffcoat, M.K., C.E. Lewis, M.S. Reddy, C.Y. Wank and M. Redford, 2000. Post-menopausal bone loss and its relationship to oral bone loss. Periodontology, 23: 94-102. PMID: 11276771

Jonasson, G., G. Bankvall and S. Kiliaridis, 2001. Estimation of skeletal bone mineral density by means of the trabecular pattern of the alveolar bone, its interdental thickness and the bone mass of the mandible. Oral Surgery, Oral Med. Oral Pathol. Oral Radiol. Endodont., 92: 346-352. DOI: $10.1067 /$ moe.2001.116494

Karayianni, K., K. Homer, A. Mitsea, L. Berkas and M. Mastoris et al., 2007. Accuracy in osteoporosis diagnosis of a combination of mandibular cortical width measurement on dental panoramic radiographs and a clinical risk index (OSIRIS): The OSTEODENT project. Bone, 40: 223-229. PMID: 16979965 
Khojastehpour, L., S.H. Shahidi, S. Barghan and E.L. Aflaki, 2009. Efficacy of panoramic mandibular index in diagnosing osteoporosis in women. J. Dentistry Tehran Univ. Med. Sci., 6: 11-15.

Klemetti, E, H.L. Collin, H. Forss, H. Markkanen and V. Lassila, 1994. Mineral status of skeleton and advanced periodontal disease. J. Clin. Periodontol., 21: 184-188. PMID: 8157771

Klemetti, E. and P. Vainio, 1993. Effect of bone mineral density in skeleton and mandible on extraction of teeth and clinical alveolar height. J. Prosthet Dent., 70: 21-25. PMID: 8366453

Krejci, C.B. and N.F. Bissada, 2002. Women's health issues and their relationship to periodontitis. J. Am. Dental Associat., 133: 323-329 PMID: 11934187

Ledgerton, D., K. Horner, H. Devlin and H. Worthington, 1999. Radiomorphometric indices of the mandible in a British female population. Dentomaxillofac. Radiol., 28: 173-181. PMID: 10740473

Ledgerton, D., K. Horner, H. Devlin and H. Worthington, 1997. Panoramic mandibular index as a radiomorphometric tool: An assessment of precision. Dentomaxillofac. Radiol., 26: 95-100. PMID: 9442624
NIH, 2004. The surgeon general's report on bone health and osteoporosis: What it means to you. NIH Turning Discovery Into Health.

OHBD, 2012. NIH Osteoporosis and Related Bone Diseases National Resource Center. Oral Health and Bone Disease.

Sapthagirivasan, V. and M. Anburajan, 2012. Heel bone mass of a young South Indian population with a Nigerian population residing in a South Indian suburban neighborhood: A comparative study. Osteoporosis Int. J., 23: 2661-2669. DOI: $10.1007 / \mathrm{s} 00198-012-1898-9$

Taguchi, A., 2010. Triage screening for osteoporosis in dental clinics using panoramic radiographs. Oral Diseases, 16: 316-327. DOI: $10.1111 / \mathrm{j} .1601-0825.2009 .01615 . x$

Taichman, R.S., 2005. Blood and bone: Two tissues whose fates are intertwined to create the hematopoietic stem-cell niche. Blood, 105: 2631-2639. PMID: 15585658 